\title{
Application of C4.5 Algorithm to Prediction Sales at PT. Sumber Sayur Segar
}

\author{
${ }^{1}$ Fadhila, ${ }^{2}$ Penda Sudarto Hasugian
}

1,2Informatics Engineering, STMIK Pelita Nusantara, Sumatera Utara, Indonesia

\begin{tabular}{l} 
Article Info \\
\hline Article history: \\
Received $01 / 12 / 2021$ \\
Revised $13 / 12 / 2021$ \\
Accepted $17 / 01 / 2022$ \\
Available online $30 / 01 / 2022$
\end{tabular}

\section{Keywords:}

C4.5 Algorithm

Data Mining

Sales Prediction

\begin{abstract}
Fresh vegetables, fruits and fresh meat are one of the basic needs for human life. The need for fresh vegetables, fruits and meat is one of the most important factors for buyers before making a purchase transaction. Likewise with the needs of fresh vegetables, fruit and meat needed by restaurants, cafes, hospitals, hotels and so on. With the increasing number of requests from customers for the needs of fresh vegetables, fruit and meat, companies engaged in the supply and sale of these necessities need to record sales transactions so that there are no stock vacancies and excess stock of goods. Therefore, companies must be more careful in providing fresh vegetables, fruits and meat which are in great demand, so it needs a data processing in the form of data mining using the C4.5 algorithm. In this study, the predicted sales transactions are the last three months of January, February and March 2021. Then for the sales prediction criteria used are in the form of price, type of goods, type of unit and month of sale so that from these criteria can be obtained sales transactions that are selling or not selling. Data mining is a process of mining important information from a very large data. While the C4.5 algorithm is a data classification that has numeric and categorical attributes, where the results of the classification process in the form of rules can be used to predict the value of discrete type attributes from new records. The system was built using the PHP programming language and $M Y S Q L$ as the database. This study obtained predictive results which were implemented in the form of a decision tree, namely the category of types of vegetables belonging to the best-selling sales transactions.
\end{abstract}

This is an open access article under the CC BY-NC license.

\section{Corresponding Author:}

Fadhila,

Informatics Engineering,

STMIK Pelita Nusantara Medan,

Jl. Iskandar Muda No. 1 Medan, 20154, Indonesia.

Email: fadhilla@gmail.com

\section{Introduction}

Along with the rapid development of information technology in the current era, it is one of the factors that advances a business field, one of which is in the field of marketing and sales. Information technology systems have been widely used in various agencies, organizations and by individuals with the aim that business activities run efficiently and accurately, so that the company's expectations for every job can be done easily and quickly. To support this, we need a system that is designed according to the needs of each company. One of them is to predict sales in the form of vegetables, fish, fruits and meat that are most needed and less needed by consumers.

To predict sales of vegetables, fish, fruits and vegetables using data mining techniques by applying the C4.5 algorithm. Data mining is the extraction of important or interesting information or patterns from existing data in large databases as a set of techniques that are used automatically to thoroughly 
explore and bring to the surface complex relationships in very large data sets (Dongoran, 2019). To implement data mining techniques in predicting sales, the $c 4.5$ algorithm is used. The C4.5 algorithm is one method for making a decision tree based on the training data that has been provided and this method is used to classify or group datasets, where the C4.5 algorithm is basically a decision tree formation (Shiddiq, Niswatin, \& Farida, 2018).

This study aims to find out how to design and build a data mining application system to predict sales, and how to find out data mining techniques by applying the c4.5 algorithm to predict sales, as well as how to find out how much the result of applying the c4.5 algorithm to predict sales of vegetables, fish, meat and fruit.

Some of the literature sources used in this study are based on previous research related to the current research topic in several journals entitled Analysis of the C4.5 Algorithm for prediction of agricultural drug sales at Dewi Sri Stores where in this study, the c4.5 algorithm is very feasible to use. in predicting sales of agricultural drugs with an accuracy rate of $75 \%$ (Dewi \& Mauladi, 2020). Then the research entitled C4.5 Algorithm Analysis To Predict Motorcycle Sales At PT. Capella Dinamik Nusantara Muka Kuning Branch, this study analyzes the pattern of sales data so that it can produce motorcycle sales predictions which will later be useful for motorcycle distribution in several regions (Azwanti, 2018). The following research is the Application of Data Mining for Wallpaper Sales Prediction Using the C4.5 Algorithm, this study predicts wallpaper sales transactions by applying the c4.5 algorithm in the form of a decision tree type data classification that classifies sales in terms of brand, color, motif, material quality, size and price (Eska, 2018). Then the research entitled AC Sales Prediction Application Using Decision Tree With C4.5 Algorithm, where the application of the c4.5 algorithm in this study as an alternative to predict the best-selling product that can be seen in the decision tree by calculating the value of gain information until the value cannot be calculated (Putri \& Waspada, 2018). Subsequent research in the journal entitled Application of Data Mining for Readymix Sales Prediction Using the C4.5 Algorithm Method at PT. Remicom Widyaprima, this research analyzes Readymix sales transactions using product quality, delivery and product warranty variables. From the results of the application of the c 4.5 algorithm, it shows that the c 4.5 algorithm is very suitable for use in predicting sales transactions (Hendra, 2020). Then research on the Application of Data Mining for Prediction of Medical Device Sales Using the C4.5 Algorithm PT. Murni Indah Sentosa. Predicting the sale of medical devices with the variables used are category of goods, price and amount sold (Fikri \& Verina, 2021). Another study entitled Prediction of Sales of Bread Products Using the C4.5 Algorithm at PT. Prima Top Catering. This study predicts sales of bakery products so that the stock is always stable with the variables used in the form of types of goods, prices, and quantities sold (Effendi \& Rahmawati, 2019). Then another study entitled Data Mining Analysis of Tire Sales Using the C4.5 Algorithm. This study predicts tire sales transactions with the aim of optimizing tire stock in the warehouse (Anggraini, Defit, \& Nurcahyo, 2018). The last research study used in this research is Product Sales Prediction Analysis Using the C4.5 Method (Case Study: PT. Kawan Lama Ace Hardware). This study predicts product sales using the c4.5 algorithm which results can be seen through a decision tree (Lubis, 2019).

\section{Method}

\subsection{Research Framework}

Research stages are steps or techniques that are arranged regularly to solve the problems to be discussed. The purpose of the research stage is to collect information or data relating to the problem to be studied. 


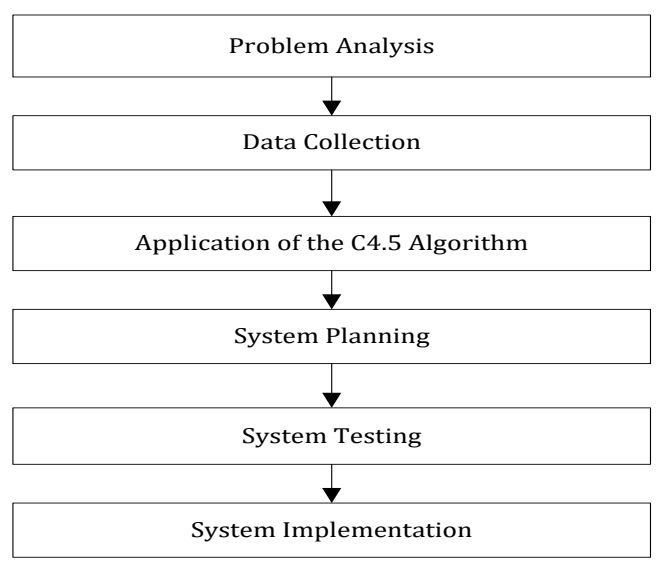

Figure 1. Research Framework

\subsection{Description of the Framework}

The description of the research framework is a further explanation of the steps in the research carried out:

a. Problem Analysis

Problem analysis is the first step carried out in this study which is divided into three types of analysis, namely:

1). Identifying Problems

To search, find, collect, research, register, record data and information needed in building sales prediction data mining applications

2). Formulating the Problem

Formulate the current problem in predicting the sales of vegetables, fish, meat and fruits with the aim of stabilizing the supply of goods every day.

3). Creating Research Objectives and Benefits

Determine what is the purpose of building a data mining application to predict sales and their benefits for the company.

b. Data Collection

Data collection is done by using R\&D (Research And Development) technique.

1). Study of Literature

The literature study used in this research is in the form of searching for library sources or references from several sources such as books, national journals, research report articles, and internet site searches.

2). Interview

Interviews were conducted to obtain information directly by asking the respondents, the respondents referred to in this study were Mr. David, B.B.A as the President Director of PT. Sumber Sayur Segar.

c. Application of the C4.5 Algorithm

This stage is an analysis or process of data that has been previously collected using the C4.5 algorithm, with the aim that the problem formulation can be resolved so that the sales prediction data mining application at PT. Sumber Sayur Segar can be useful.

d. System Planning

System design is a stage to describe the system to be built.

1). System Modeling with UML

The process of building or forming a model of a real system in a particular formal language. To design the system using use case diagrams, activity diagrams, and class diagrams.

2). System Design 
The stages of this system design are describing the system to be built in the form of main page design, input menu design and output menu design.

e. System Testing

This stage is testing the functionality of all systems and error handling on the system made whether it is as expected. Testing this system will be first carried out on the localhost server, for example: http://localhost/predik-penjualan-algoritma-c45/.

f. System Implementation

This stage is the stage of putting the system so that it is ready for operation and can be seen as an effort to realize the system that has been designed. The steps in this implementation phase are the sequence of activities from start to finish that must be carried out in realizing the system that has been designed.

\section{Results and Discussion}

\subsection{Analysis C4.5 Algorithm}

The following is an analysis of sales of vegetables, fish, meat and fruit at PT. Sumber Sayur Segar period January-March 2021.

a. Select Root Attribute

To facilitate the explanation of the C4.5 Algorithm, it is necessary to determine the root attribute based on the highest gain value for each attribute.

TABLE 1.

Sales Data Analysis With C4.5 Algorithm

\begin{tabular}{|c|c|c|c|c|c|c|}
\hline Code & Name of Goods & Unit Type & Types of Goods & Month/Year & Price (IDR) & Conclusion \\
\hline KTG & Potato & $\mathrm{Kg}$ & Fruit & Mar-21 & 1.470 .000 & Selling \\
\hline SWJ & Mustard Greens & $\mathrm{Kg}$ & Vegetable & Mar-21 & 480.000 & Selling \\
\hline SAP & Chinese & $\mathrm{Kg}$ & Vegetable & Jan-21 & 320.000 & Selling \\
\hline $\mathrm{KPH}$ & White cabbage & $\mathrm{Kg}$ & Vegetable & Feb-21 & 518.000 & Selling \\
\hline TMT & Tomatoes & $\mathrm{Kg}$ & Fruit & Jan-21 & 630.000 & Selling \\
\hline TMN & Cucumber & $\mathrm{Kg}$ & Fruit & Jan-21 & 292.000 & Selling \\
\hline DSP & Celery & $\mathrm{Kg}$ & Vegetable & Jan-21 & 396.000 & Selling \\
\hline DPE & Prey Leaves & $\mathrm{Kg}$ & Vegetable & Mar-21 & 560.000 & Not Selling \\
\hline SLD & Lettuce & $\mathrm{Kg}$ & Vegetable & Mar-21 & 666.000 & Selling \\
\hline BMB & Onion Bombay & $\mathrm{Kg}$ & Vegetable & Feb-21 & 600.000 & Not Selling \\
\hline CMP & Red Chili & $\mathrm{Kg}$ & Vegetable & Feb-21 & 1.575 .000 & Not Selling \\
\hline CHP & Green Chili & $\mathrm{Kg}$ & Vegetable & Mar-21 & 1.064 .000 & Selling \\
\hline JMT & Old Ginger & $\mathrm{Kg}$ & Vegetable & Jan-21 & 224.000 & Selling \\
\hline WTL & Carrot & $\mathrm{Kg}$ & Vegetable & Mar-21 & 210.000 & Not Selling \\
\hline JGK & Peeled Corn & $\mathrm{Kg}$ & Fruit & Jan-21 & 200.000 & Selling \\
\hline PSK & Banana Kepok & SSR & Fruit & Feb-21 & 270.000 & Not Selling \\
\hline PSB & Banana & SSR & Fruit & Jan-21 & 700.000 & Selling \\
\hline JKN & Lime & $\mathrm{Kg}$ & Fruit & Jan-21 & 84.000 & Not Selling \\
\hline JKM & Honey Orange & $\mathrm{Kg}$ & Fruit & Jan-21 & 750.000 & Selling \\
\hline MLM & Melon & $\mathrm{Kg}$ & Fruit & Mar-21 & 375.000 & Not Selling \\
\hline SMK & Watermelon & $\mathrm{Kg}$ & Fruit & Mar-21 & 390.000 & Not Selling \\
\hline ALP & Avocado & $\mathrm{Kg}$ & Fruit & Mar-21 & 448.000 & Not Selling \\
\hline $\mathrm{BKG}$ & Jicama & $\mathrm{Kg}$ & Fruit & Mar-21 & 110.000 & Selling \\
\hline $\mathrm{AMF}$ & Red Apple & $\mathrm{Kg}$ & Fruit & Feb-21 & 320.000 & Not Selling \\
\hline BRS & Rice & $\mathrm{Kg}$ & Basic Food & Jan-21 & 5.600 .000 & Selling \\
\hline $\mathrm{MGCH}$ & Cooking Oil & $\mathrm{Kg}$ & Basic Food & Jan-21 & 1.800 .000 & Selling \\
\hline $\mathrm{BKH}$ & White Rice & $\mathrm{Kg}$ & Basic Food & Jan-21 & 200.000 & Selling \\
\hline BKP & Black Stiky Rice & $\mathrm{Kg}$ & Basic Food & Mar-21 & 340.000 & Selling \\
\hline GUP & Sugar & $\mathrm{Kg}$ & Basic Food & Jan-21 & 1.120 .000 & Selling \\
\hline TAE & Chicken Eggs & PPN & Basic Food & Jan-21 & 3.510 .000 & Selling \\
\hline
\end{tabular}




\begin{tabular}{clccccc}
\hline Code & Name of Goods & Unit Type & Types of Goods & Month/Year & Price (IDR) & Conclusion \\
\hline TET & Wheat & Kg & Basic Food & Mar-21 & 50.000 & Not Selling \\
MTG & Butter & Kg & Basic Food & Mar-21 & 255.000 & Selling \\
KPS & Cheese Prochis & PACK & Basic Food & Feb-21 & 160.000 & Selling \\
TPY & Quail eggs & BTR & Basic Food & Mar-21 & 9.000 & Not Selling \\
AYT & Whole Chicken & Kg & Meat & Mar-21 & 40.160 .000 & Selling \\
ATG & Broilers & Kg & Meat & Jan-21 & 3.132 .500 & Selling \\
AKS & Kalasan Chicken & Kg & Meat & Jan-21 & 576.000 & Not Selling \\
AYK & Kampong & Kg & Meat & Jan-21 & 1.156 .000 & Not Selling \\
TLA & Chicken Bone & Kg & Meat & Feb-21 & 36.000 & Not Selling \\
CKA & Chicken Feet & Kg & Meat & Feb-21 & 360.000 & Selling \\
LMKA & Chicken fat & Kg & Meat & Jan-21 & 14.000 & Not Selling \\
HAM & Gizzar Heart & Kg & Meat & Feb-21 & 56.000 & Not Selling \\
FDD & Breast Filler & Kg & Meat & Mar-21 & 5.225 .500 & Selling \\
FPH & Thigh Fillers & Kg & Meat & Jan-21 & 1.182 .000 & Not Selling \\
DKG & Lamb & Kg & Meat & Jan-21 & 665.500 & Not Selling \\
DSA & Beef & Kg & Meat & Feb-21 & 13.585 .000 & Selling \\
DHD & Special Meat in & Kg & Meat & Feb-21 & 10.125 .000 & Selling \\
OXT & Oxtail & Kg & Meat & Feb-21 & 5.500 .000 & Selling \\
IGAS & Beef Ribs & Kg & Meat & Jan-21 & 660.000 & Not Selling \\
SAF & Fiesta Chicken & BKS & Meat & Feb-21 & 3.984 .000 & Selling \\
BFFLS & Buffalo & Kg & Meat & Feb-21 & 1.658 .000 & Selling \\
PBBI & Pork Ribs & Kg & Meat & Feb-21 & 3.835 .000 & Selling \\
CKTBI & Pork Chop & Kg & Meat & Mar-21 & 1.625 .000 & Selling \\
DKPP & Crab Meat & PACK & Fish & Feb-21 & 870.000 & Selling \\
\hline
\end{tabular}

b. Create a Branch for Each Case

TABLE 2.

Node 1 Calculation For Price

\begin{tabular}{|c|c|c|c|c|c|c|c|}
\hline NODE & Attribute & Attribute Value & $\begin{array}{c}\text { Amount Case } \\
\text { (S) }\end{array}$ & $\begin{array}{l}\text { Selling } \\
\text { (S1) }\end{array}$ & $\begin{array}{l}\text { Not Selling } \\
\text { (S2) }\end{array}$ & Entropy & Gain \\
\hline \multirow[t]{28}{*}{1} & Total & Total & 55 & 35 & 20 & 0.9457 & \multirow{6}{*}{0.0353} \\
\hline & \multirow[t]{10}{*}{ Types of Goods } & Vegetables & 11 & 7 & 4 & 0.9457 & \\
\hline & & Fruit & 14 & 8 & 6 & 0.9852 & \\
\hline & & Meat & 19 & 11 & 8 & 0.9819 & \\
\hline & & Fish & 1 & 1 & 0 & 0 & \\
\hline & & Basic of Foods & 10 & 8 & 2 & 0.7219 & \\
\hline & & Bks & 1 & 1 & 0 & 0 & \\
\hline & & Pack & 2 & 2 & 0 & 0 & \\
\hline & & Ppn & 1 & 1 & 0 & 0 & 0.0764 \\
\hline & & $\mathrm{Btr}$ & 1 & 0 & 1 & 0 & \\
\hline & & SSR & 2 & 1 & 1 & 1 & \\
\hline & \multirow[t]{3}{*}{ Month/Year } & Jan/2021 & 22 & 15 & 7 & 0.9024 & \multirow{3}{*}{0.0076} \\
\hline & & Feb/2021 & 17 & 11 & 6 & 0.9367 & \\
\hline & & Mar/2021 & 16 & 9 & 7 & 0.9887 & \\
\hline & \multirow[t]{14}{*}{ Price (IDR) } & 9000 & 1 & 0 & 1 & 0 & \multirow{14}{*}{0.873} \\
\hline & & 14000 & 1 & 0 & 1 & 0 & \\
\hline & & 36000 & 1 & 0 & 1 & 0 & \\
\hline & & 50000 & 1 & 0 & 1 & 0 & \\
\hline & & 56000 & 1 & 0 & 1 & 0 & \\
\hline & & 84000 & 1 & 0 & 1 & 0 & \\
\hline & & 110000 & 1 & 1 & 0 & 0 & \\
\hline & & 160000 & 1 & 1 & 0 & 0 & \\
\hline & & 200000 & 2 & 2 & 0 & 0 & \\
\hline & & 210000 & 1 & 0 & 1 & 0 & \\
\hline & & 224000 & 1 & 1 & 0 & 0 & \\
\hline & & 255000 & 1 & 1 & 0 & 0 & \\
\hline & & 480000 & 1 & 1 & 0 & 0 & \\
\hline & & 270000 & 1 & 0 & 1 & 0 & \\
\hline
\end{tabular}




\begin{tabular}{|c|c|c|c|c|c|c|c|}
\hline NODE & Attribute & Attribute Value & $\begin{array}{c}\text { Amount Case } \\
\text { (S) }\end{array}$ & $\begin{array}{l}\text { Selling } \\
\text { (S1) }\end{array}$ & $\begin{array}{l}\text { Not Selling } \\
\text { (S2) }\end{array}$ & Entropy & Gain \\
\hline & & 292000 & 1 & 1 & 0 & 0 & \\
\hline & & 320000 & 2 & 1 & 1 & 1 & \\
\hline & & 340000 & 1 & 1 & 0 & 0 & \\
\hline & & 375000 & 1 & 0 & 1 & 0 & \\
\hline & & 390000 & 1 & 0 & 1 & 0 & \\
\hline & & 396000 & 1 & 1 & 0 & 0 & \\
\hline & & 448000 & 2 & 1 & 1 & 1 & \\
\hline & & 518000 & 1 & 1 & 0 & 0 & \\
\hline & & 560000 & 1 & 0 & 1 & 0 & \\
\hline & & 576000 & 1 & 0 & 1 & 0 & \\
\hline & & 600000 & 1 & 0 & 1 & 0 & \\
\hline & & 630000 & 1 & 1 & 0 & 0 & \\
\hline & & 660000 & 1 & 0 & 1 & 0 & \\
\hline & & 665000 & 0 & 0 & 0 & 0 & \\
\hline & & 666000 & 1 & 1 & 0 & 0 & \\
\hline & & 700000 & 1 & 1 & 0 & 0 & \\
\hline & & 750000 & 1 & 1 & 0 & 0 & \\
\hline & & 870000 & 1 & 1 & 0 & 0 & \\
\hline & & 1064000 & 1 & 1 & 0 & 0 & \\
\hline & & 1120000 & 1 & 1 & 0 & 0 & \\
\hline & & 1182500 & 1 & 0 & 1 & 0 & \\
\hline & & 1575000 & 1 & 0 & 1 & 0 & \\
\hline & & 1625000 & 1 & 1 & 0 & 0 & \\
\hline & & 1800000 & 1 & 1 & 0 & 0 & \\
\hline & & 3152500 & 0 & 0 & 0 & 0 & \\
\hline & & 3510500 & 0 & 0 & 0 & 0 & \\
\hline & & 3835000 & 1 & 1 & 0 & 0 & \\
\hline & & 5500000 & 1 & 1 & 0 & 0 & \\
\hline & & 5600000 & 1 & 1 & 0 & 0 & \\
\hline & & 10125000 & 1 & 1 & 0 & 0 & \\
\hline & & 13595000 & 0 & 0 & 0 & 0 & \\
\hline & & 40160000 & 1 & 1 & 0 & 0 & \\
\hline
\end{tabular}

c. Calculating Entrophy and Gain Values for Node 1

a. Node 1 Calculation for Total

$$
\begin{aligned}
\text { Entropy(Total) }= & \left(-\frac{\mathrm{S}_{1}}{\mathrm{~S}} * \log _{2}\left(\frac{\mathrm{S}_{1}}{\mathrm{~S}}\right)\right)+\left(-\frac{\mathrm{S}_{2}}{\mathrm{~S}} * \log _{2}\left(\frac{\mathrm{S}_{2}}{\mathrm{~S}}\right)\right)=\left(-\frac{35}{55} * \log _{2}\left(\frac{35}{55}\right)\right)+\left(-\frac{20}{55} * \log _{2}\left(\frac{20}{55}\right)\right)=0.4150 \\
& +0.5307=0.9457
\end{aligned}
$$

b. Entropy Calculation

- Entropy Types of Goods

Entropy(Total,Vegetables) $=\left(-\frac{7}{11} * \log _{2}\left(\frac{7}{11}\right)\right)+\left(\frac{4}{11} * \log _{2}\left(\frac{4}{11}\right)\right)=0.9457$

Entropy(Total,Fruits) $=\left(-\frac{8}{14} * \log _{2}\left(\frac{8}{14}\right)\right)+\left(\frac{6}{14} * \log _{2}\left(\frac{6}{14}\right)\right)=0.9852$

Entropy(Total,Meat) $=\left(-\frac{11}{19} * \log _{2}\left(\frac{11}{19}\right)\right)+\left(\frac{8}{19} * \log _{2}\left(\frac{8}{19}\right)\right)=0.9819$

Entropy(Total,Fish) $=\left(-\frac{1}{1} * \log _{2}\left(\frac{1}{1}\right)\right)+\left(\frac{0}{1} * \log _{2}\left(\frac{0}{1}\right)\right)=0$

Entropy(Total,Basic of Food) $=\left(-\frac{8}{10} * \log _{2}\left(\frac{8}{10}\right)\right)+\left(\frac{2}{10} * \log _{2}\left(\frac{2}{10}\right)\right)=0.7219$

- Entropy Unit

Entropy $($ Total, $\mathrm{Kg})=\left(-\frac{30}{48} * \log _{2}\left(\frac{30}{48}\right)\right)+\left(\frac{18}{48} * \log _{2}\left(\frac{18}{48}\right)\right)=0.9544$

Entropy(Total,Bks) $=\left(-\frac{1}{1} * \log _{2}\left(\frac{1}{1}\right)\right)+\left(\frac{0}{1} * \log _{2}\left(\frac{0}{1}\right)\right)=0$

Entropy (Total,Pack) $=\left(-\frac{2}{2} * \log _{2}\left(\frac{2}{2}\right)\right)+\left(\frac{0}{2} * \log _{2}\left(\frac{0}{2}\right)\right)=0$ 
Entropy $($ Total,Ppn $)=\left(-\frac{1}{1} * \log _{2}\left(\frac{1}{1}\right)\right)+\left(\frac{0}{2} * \log _{2}\left(\frac{0}{2}\right)\right)=0$

Entropy (Total,Btr $)=\left(-\frac{0}{1} * \log _{2}\left(\frac{0}{1}\right)\right)+\left(\frac{1}{1} * \log _{2}\left(\frac{1}{1}\right)\right)=0$

Entropy(Total,SSR $)=\left(-\frac{1}{2} * \log _{2}\left(\frac{1}{2}\right)\right)+\left(\frac{1}{2} * \log _{2}\left(\frac{1}{2}\right)\right)=1$

- Entropy Month/Year

Entropy $\left(\right.$ Total,Jan/2021) $=\left(-\frac{15}{22} * \log _{2}\left(\frac{15}{22}\right)\right)+\left(\frac{7}{22} * \log _{2}\left(\frac{7}{22}\right)\right)=0.9024$

Entropy (Total,Feb/2021) $=\left(-\frac{11}{17} * \log _{2}\left(\frac{11}{17}\right)\right)+\left(\frac{6}{17} * \log _{2}\left(\frac{6}{17}\right)\right)=0.9367$

Entropy $($ Total,Mar $/ 2021)=\left(-\frac{9}{16} * \log _{2}\left(\frac{9}{16}\right)\right)+\left(\frac{7}{16} * \log _{2}\left(\frac{7}{16}\right)\right)=0.9887$

- Entropy Price (IDR)

Entropy $\left(\right.$ Total,9000) $=\left(-\frac{0}{1} * \log _{2}\left(\frac{0}{1}\right)\right)+\left(\frac{1}{1} * \log _{2}\left(\frac{1}{1}\right)\right)=0$

Entropy $($ Total, 14000$)=\left(-\frac{0}{1} * \log _{2}\left(\frac{0}{1}\right)\right)+\left(\frac{1}{1} * \log _{2}\left(\frac{1}{1}\right)\right)=0$

Entropy $\left(\right.$ Total,36000) $=\left(-\frac{0}{1} * \log _{2}\left(\frac{0}{1}\right)\right)+\left(\frac{1}{1} * \log _{2}\left(\frac{1}{1}\right)\right)=0$

Entropy $($ Total, 56000$)=\left(-\frac{0}{1} * \log _{2}\left(\frac{0}{1}\right)\right)+\left(\frac{1}{1} * \log _{2}\left(\frac{1}{1}\right)\right)=0$

Entropy $($ Total, 480000$)=\left(-\frac{1}{1} * \log _{2}\left(\frac{1}{1}\right)\right)+\left(\frac{0}{1} * \log _{2}\left(\frac{0}{1}\right)\right)=0$

Entropy $($ Total, 320000$)=\left(-\frac{1}{2} * \log _{2}\left(\frac{1}{2}\right)\right)+\left(\frac{1}{2} * \log _{2}\left(\frac{1}{2}\right)\right)=1$

Entropy $($ Total, 375000$)=\left(-\frac{0}{1} * \log _{2}\left(\frac{0}{1}\right)\right)+\left(\frac{1}{1} * \log _{2}\left(\frac{1}{1}\right)\right)=0$

Entropy $($ Total, 390000$)=\left(-\frac{0}{1} * \log _{2}\left(\frac{0}{1}\right)\right)+\left(\frac{1}{1} * \log _{2}\left(\frac{1}{1}\right)\right)=0$

Entropy $($ Total, 448000$)=\left(-\frac{1}{2} * \log _{2}\left(\frac{1}{2}\right)\right)+\left(\frac{1}{2} * \log _{2}\left(\frac{1}{2}\right)\right)=1$

Entropy $($ Total, 518000$)=\left(-\frac{1}{1} * \log _{2}\left(\frac{1}{1}\right)\right)+\left(\frac{0}{1} * \log _{2}\left(\frac{0}{1}\right)\right)=0$

Entropy $($ Total, 560000$)=\left(-\frac{0}{1} * \log _{2}\left(\frac{0}{1}\right)\right)+\left(\frac{1}{1} * \log _{2}\left(\frac{1}{1}\right)\right)=0$

Entropy $\left(\right.$ Total, 666000) $=\left(-\frac{1}{1} * \log _{2}\left(\frac{1}{1}\right)\right)+\left(\frac{0}{1} * \log _{2}\left(\frac{0}{1}\right)\right)=0$

Entropy $\left(\right.$ Total,700000) $=\left(-\frac{1}{1} * \log _{2}\left(\frac{1}{1}\right)\right)+\left(\frac{0}{1} * \log _{2}\left(\frac{0}{1}\right)\right)=0$

Entropy $($ Total, 750000$)=\left(-\frac{1}{1} * \log _{2}\left(\frac{1}{1}\right)\right)+\left(\frac{0}{1} * \log _{2}\left(\frac{0}{1}\right)\right)=0$

Entropy $($ Total, 1470000$)=\left(-\frac{1}{1} * \log _{2}\left(\frac{1}{1}\right)\right)+\left(\frac{0}{1} * \log _{2}\left(\frac{0}{1}\right)\right)=0$

Entropy $($ Total, 1575000$)=\left(-\frac{0}{1} * \log _{2}\left(\frac{0}{1}\right)\right)+\left(\frac{1}{1} * \log _{2}\left(\frac{1}{1}\right)\right)=0$

Entropy $($ Total, 1625000$)=\left(-\frac{1}{1} * \log _{2}\left(\frac{1}{1}\right)\right)+\left(\frac{0}{1} * \log _{2}\left(\frac{0}{1}\right)\right)=0$

Entropy $($ Total, 1658000$)=\left(-\frac{1}{1} * \log _{2}\left(\frac{1}{1}\right)\right)+\left(\frac{0}{1} * \log _{2}\left(\frac{0}{1}\right)\right)=0$

Entropy $($ Total, 3152500$)=\left(-\frac{0}{1} * \log _{2}\left(\frac{0}{1}\right)\right)+\left(\frac{1}{1} * \log _{2}\left(\frac{1}{1}\right)\right)=0$

Entropy $\left(\right.$ Total,3510500) $=\left(-\frac{0}{1} * \log _{2}\left(\frac{0}{1}\right)\right)+\left(\frac{1}{1} * \log _{2}\left(\frac{1}{1}\right)\right)=0$

Entropy $($ Total, 2984000$)=\left(-\frac{0}{1} * \log _{2}\left(\frac{0}{1}\right)\right)+\left(\frac{1}{1} * \log _{2}\left(\frac{1}{1}\right)\right)=0$

Entropy $($ Total, 40160000$)=\left(-\frac{0}{1} * \log _{2}\left(\frac{0}{1}\right)\right)+\left(\frac{1}{1} * \log _{2}\left(\frac{1}{1}\right)\right)=0$

c. Gain Calculation

- Calculation of Information Gain Value for Items 
Gain $($ Total,Types of Food $)=0.9457-\left(\frac{11}{55} * 0.9457\right)-\left(\frac{14}{55} * 0.9852\right)\left(\frac{19}{55} * 0.9819\right)-\left(\frac{1}{55} * 0\right)-\left(\frac{0}{55} * 0\right)$

$$
-\left(\frac{10}{55} * 0.7219\right)=\mathbf{0 . 0 3 5 3}
$$

- Calculation of Information Gain Value for Unit Type

Gain(Total,Unit Type $)=0.9457-\left(\frac{48}{55} * 0.9544\right)-\left(\frac{1}{55} * 0\right)-\left(\frac{2}{55} * 0\right)-\left(\frac{0}{55} * 1\right)-\left(\frac{1}{55} * 0\right)-\left(\frac{1}{55} * 0\right)-$

$$
\left(\frac{2}{55} * 1\right)=\mathbf{0 . 0 7 6 4}
$$

- Calculation of Information Gain Value for Month/Year

Gain(Total,Month/Year) $=0.9457-\left(\frac{22}{55} * 0.9024\right)-\left(\frac{17}{55} * 0.9367\right)-\left(\frac{16}{55} * 0.9887\right)=\mathbf{0 . 0 0 7 6}$

- Calculation of Information Gain Value for Price (IDR)

Gain $($ Total,Price(IDR) $)=0.9457-\left(\frac{1}{55} * 0\right)-\left(\frac{1}{55} * 0\right)-\left(\frac{1}{55} * 0\right)-\left(\frac{1}{55} * 0\right)-\left(\frac{1}{55} * 0\right)-\left(\frac{1}{55} * 0\right)-\left(\frac{1}{55} * 0\right)$

$$
\begin{aligned}
& \text { - }\left(\frac{1}{55} * 0\right)-\left(\frac{2}{55} * 0\right)-\left(\frac{1}{55} * 0\right)-\left(\frac{1}{55} * 0\right)-\left(\frac{1}{55} * 0\right)-\left(\frac{1}{55} * 0\right)-\left(\frac{1}{55} * 0\right)-\left(\frac{1}{55} * 0\right)-\left(\frac{2}{55} * 1\right)-\left(\frac{1}{55} * 0\right) \text { - } \\
& \left(\frac{1}{55} * 0\right)-\left(\frac{1}{55} * 0\right)-\left(\frac{1}{55} * 0\right)-\left(\frac{2}{55} * 1\right)-\left(\frac{1}{55} * 0\right)-\left(\frac{1}{55} * 0\right)-\left(\frac{1}{55} * 0\right)-\left(\frac{1}{55} * 0\right)-\left(\frac{1}{55} * 0\right)-\left(\frac{1}{55} * 0\right) \\
& \text { - }\left(\frac{0}{55} * 0\right)-\left(\frac{1}{55} * 0\right)-\left(\frac{1}{55} * 0\right)-\left(\frac{1}{55} * 0\right)-\left(\frac{1}{55} * 0\right)-\left(\frac{1}{55} * 0\right)-\left(\frac{1}{55} * 0\right)-\left(\frac{1}{55} * 0\right)-\left(\frac{1}{55} * 0\right)-\left(\frac{1}{55} * 0\right) \text { - } \\
& \left(\frac{1}{55} * 0\right)-\left(\frac{1}{55} * 0\right)-\left(\frac{1}{55} * 0\right)-\left(\frac{1}{55} * 0\right)-\left(\frac{0}{55} * 0\right)-\left(\frac{0}{55} * 0\right)-\left(\frac{1}{55} * 0\right)-\left(\frac{0}{55} * 0\right)-\left(\frac{1}{55} * 0\right)-\left(\frac{1}{55} * 0\right) \\
& -\left(\frac{1}{55} * 0\right)-\left(\frac{1}{55} * 0\right)-\left(\frac{0}{55} * 0\right)-\left(\frac{1}{55} * 0\right)=\mathbf{0 . 8 7 3}
\end{aligned}
$$

\subsection{System Design}

a. Use Case Diagram

Use Case Diagram aims to find out what are the functions that exist in the data mining system to predict sales at PT. Sumber Sayur Segar uses the c4.5 algorithm which is shown below:

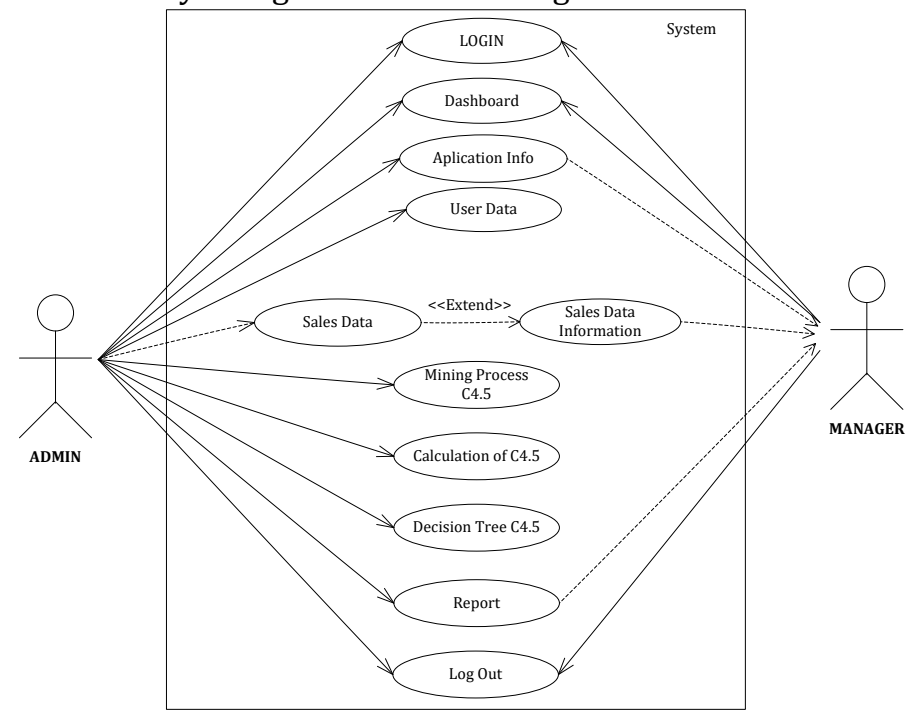

Figure 2. Use Case Diagram

\section{b. Output Result}

After designing the system, the system can be implemented from the output predictions of sales at PT. Sumber Sayur Segar uses Algorithm c4.5 as shown in the following image: 


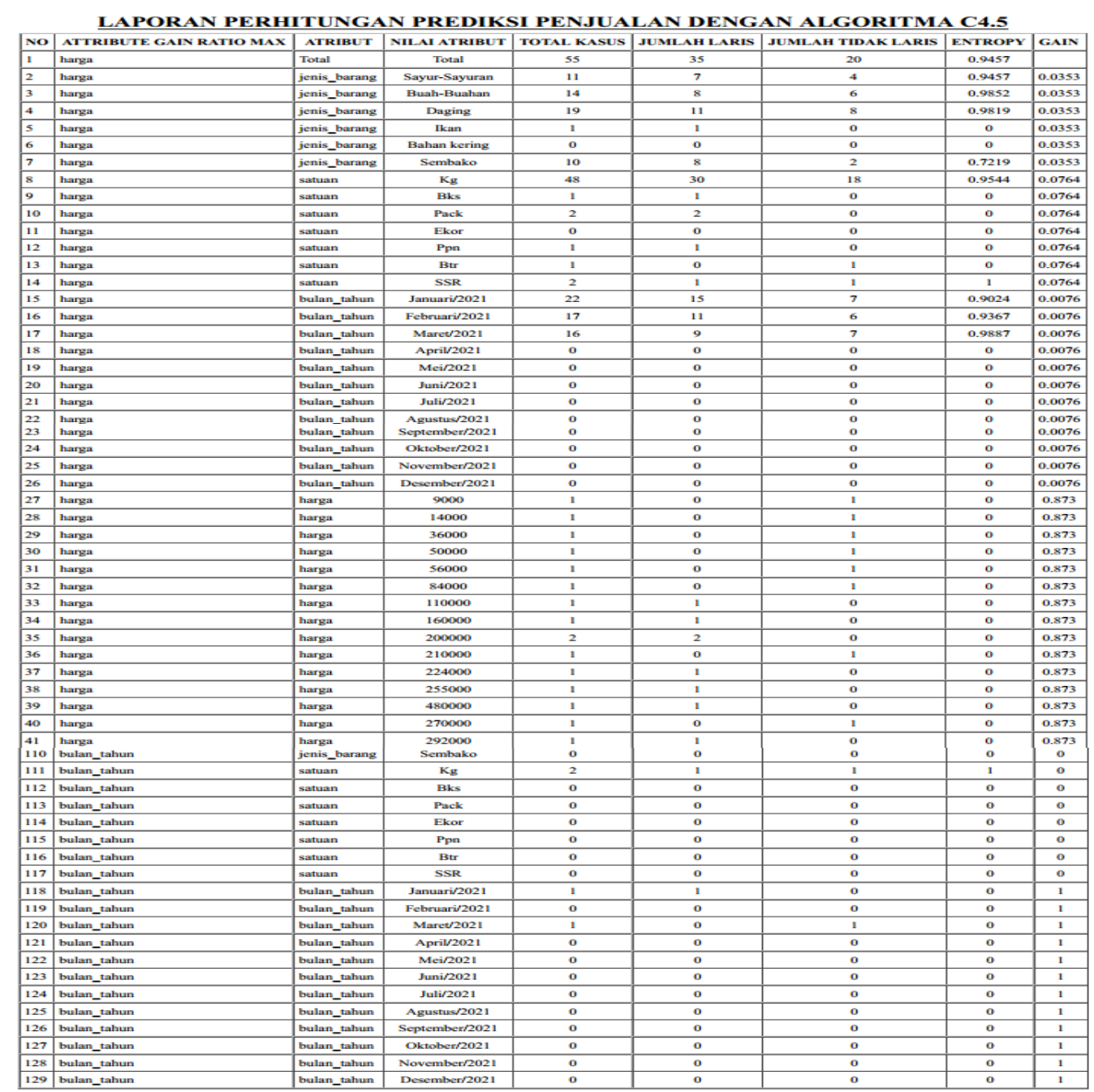

Figure 3. Output Result

\section{Conclusion}

Data mining application system to predict sales using the C4.5 Algorithm at PT. XXX is designed by using UML design or modeling using use case diagrams, activity diagrams and class diagrams, and is built using the PHP programming language and MySQL database. 4. With an application system to predict sales that applies the C4.5 Algorithm at PT. XXX, it can be seen how much sales prediction results are obtained which can be implemented in a decision tree, where the decision tree can be seen that the category of vegetable goods belongs to the sales transaction of the best-selling types of goods.

\section{References}

Anggraini, S., Defit, S., \& Nurcahyo, G. W. (2018). Analisis Data Mining Penjualan Ban Menggunakan Algoritma C4. 5. Jurnal Ilmu Teknik Elektro Komputer Dan Informatika (JITEKI), 4(2), 136.

Azwanti, N. (2018). Analisa Algoritma C4. 5 Untuk Memprediksi Penjualan Motor Pada Pt. Capella Dinamik Nusantara Cabang Muka Kuning. Inform. Mulawarman J. Ilm. Ilmu Komput, 13(1), 33.

Dewi, K. R., \& Mauladi, K. F. (2020). Analisa Algoritma C4. 5 untuk Prediksi Penjualan Obat Pertanian di Toko Dewi Sri. Prosiding SEMNAS INOTEK (Seminar Nasional Inovasi Teknologi), 4(3), 109-114.

Dongoran, N. S. (2019). PENERAPAN ALGORITMA APRIORI DAN ECONOMIC ORDER QUANTITY UNTUK PENGENDALIAN PERSEDIAN PRODUK DI ALMIRA KIDS. Universitas Islam Negeri Sultan Syarif Kasim Riau. 
Effendi, M. M., \& Rahmawati, D. (2019). Prediksi Penjualan Produk Roti Menggunakan Algoritma C4. 5 Pada PT. Prima Top Boga. Jurnal SIGMA, 9(1), 51-66.

Eska, J. (2018). Penerapan Data Mining Untuk Prediksi Penjualan Wallpaper Menggunakan Algoritma C4. 5.

Fikri, A., \& Verina, W. (2021). PENERAPAN DATA MINING UNTUK PREDIKSI PENJUALAN ALAT MEDIS MENGGUNAKAN ALGORITMA C4. 5 PT. MURNI INDAH SENTOSA. INFOSYS (INFORMATION SYSTEM) JOURNAL, 5(1), 70-83.

Hendra, H. (2020). Penerapan Data Mining untuk Prediksi Penjualan Readymix Menggunakan Metode Algoritma C4. 5 pada PT Remicon Widyaprima. Prodi Sistem Informasi.

Lubis, M. R. (2019). Analisa Prediksi Penjualan Produk Dengan Menggunakan Metode C4. 5 (Studi Kasus: PT. Kawan Lama Ace Hardware). JURIKOM (Jurnal Riset Komputer), 6(5), 545-549.

Putri, R. P. S., \& Waspada, I. (2018). Penerapan Algoritma C4. 5 pada Aplikasi Prediksi Kelulusan Mahasiswa Prodi Informatika. Khazanah Informatika: Jurnal Ilmu Komputer Dan Informatika, 4(1), 1-7.

Shiddiq, A., Niswatin, R. K., \& Farida, I. N. (2018). Ahmad Shiddiq Analisa Kepuasan Konsumen Menggunakan Klasifikasi Decision Tree Di Restoran Dapur Solo (Cabang Kediri). Generation Journal, 2(1), 9-18. 